\title{
Classification of evolutoids and pedaloids in Minkowski space-time plane
}

\author{
A. A. Abdel-Salam \\ Faculty of Science \\ Northern Border University \\ Mathematics Department \\ Saudi Arabia \\ assem.mahmoud@nbu.edu.sa
}

\author{
M. KHALIFA SAAD \\ Faculty of Science, Sohag University \\ Mathematics Department \\ EGYPT \\ Faculty of Science, Islamic University of Madinah \\ Mathematics Department \\ Saudi Arabia
}

Abstract: In this paper, we study the families of relatives of pedals and evolutes in the Minkowski space-
time plane $\mathbb{R}_{1}^{2}$. We obtain some relationships between these families which turn out to be different from
Fuclideanplane. Also.weclassifyandgeneralizethesengtionstothecategoryoffrontalcuryesin $\mathbb{R}^{2}$
Finally, some computational examples in support of our main resutsare given and plotted. Key-Words:

Minkowskispace-time,planecurve,evolute,evolutoids,pedal,pedaloids.

Received: September 26, 2020. Revised: March 2, 2021. Accepted: March 15, 2021. Published: April 10, 2021.

\section{Introduction}

At the beginning of the twentieth century, A. Einstein's theory opened a door to use of new geometries. One of them, Minkowski space-time which is simultaneously the geometry of special relativity. The geometry induced on each fixed tangent space of an arbitrary Lorentzian manifold and some of the classical differential geometry topics were introduced and treated by some researchers (see for instance, 1, 2|). Recently, the theory of degenerate submanifolds has been extended to Lorentz manifolds 3 .

A Minkowski space-time plane $\mathbb{R}_{1}^{2}$ is pseudoEuclidean plane, i.e., there are three types of directions; the spacelike, timelike and lightlike directions. The unit ball in such a plane consists of two conjugate hyperbolas with lightlike asymptotes. Many authors discussed this space from the relativity point of view with some mathematical concepts (see for more details 3 - 5 ).

In the classical differential geometry, the notions of pedal curves of regular curves in the Euclidean plane or Euclidean 3-space are classical topics.

Pedal curves belong to curves associated with the given ones by means of some geometrical construction, as evolutes, involutes, parallels, etc. These curves were introduced by well-known scotch mathematician Collin MacLaurin as locus of the foot of the perpendicular from the given point to the tangent to a given curve in the early of 18 th century. In 6, T. Nishimura gave the concept and the classification of the singularities of pedal curves of regular curves in the unit sphere. Also, Božek and Foltán have discussed the connections between singular points of the pedal curves and inflexion points of regular curves in the Euclidean plane 7 .

Unfortunately, if the curve is not regular at some points, then as the classical way, we cannot define the pedal curve at this point. T. Fukunaga and M. Takahashi studied frontals (or fronts) in Euclidean plane and Legendrian curves (or Legendrian immersions) in the unit tangent bundle of $\mathbb{R}^{2}$ (see [8-10]). The differential geometric properties of the frontal are studied in 11, 12. $\mathrm{Li}$ and D. Pei defined the pedal curves of fronts and gave the classification of singularities of the pedal curves of fronts in the sphere. The most difference between a regular curve and a frontal is that the frontal might exist singular points. A key tool for studying of the frontal is so called moving frame defined in the unit tangent bundle.

In this paper, by using the moving frame, we give a new definition of pedal curve of the frontal in the Minkowski space-time plan. We remark that this new definition of the pedal curve is consistent with the classical one when the curve is a regular curve. 
Evolute curve is defined to be the envelope along the curve $\zeta$ of the family of normal lines. The envelope of the tangent line family along $\zeta$ is the original curve of the tangent line at each $\zeta$ curve's inflection point together. The definition of evolutoids was introduced by Giblin and Warder as a one-parameter family of associated curves of $\zeta$, which fills in the gap between the evolute and the original curve [13. In addition, the envelope of the family of lines is defined as each member of the evolutoids, so that each line has a constant angle with the tangent line at the same point of the original curve.

Similar to the evolutoids, we introduce the concept of pedaloids which are a family of relatives of a pedal curve. Also, we obtain a relationship between the pedaloids and evolutoids of a regular curve and use this relationship to investigate frontal curves.

During this paper, we assume that the curve $\zeta$ is a timelike curve and all maps are class $C^{\infty}$ unless the contrary is explicitly stated.

\section{Differential Geometric Prop- erties}

The Minkowski plane $\mathbb{R}_{1}^{2}$ is the plane $\mathbb{R}^{2}$ endowed with the metric induced by the pseudoscalar product $\langle\mathbf{x}, \mathbf{y}\rangle=-x_{1} y_{1}+x_{2} y_{2}$, where $\mathbf{x}=\left(x_{1}, x_{2}\right)$ and $\mathbf{y}=\left(y_{1}, y_{2}\right)$. We say that, a vector $\mathbf{x}$ in $\mathbb{R}_{1}^{2}$ is spacelike, lightlike or timelike if $\langle\mathbf{x}, \mathbf{x}\rangle>0,\langle\mathbf{x}, \mathbf{x}\rangle=0$ or $\langle\mathbf{x}, \mathbf{x}\rangle<0$, respectively. The norm of a vector $\mathbf{x}=\left(x_{1}, x_{2}\right) \in \mathbb{R}_{1}^{2}$ is defined by $\|\mathbf{x}\|=\sqrt{|\langle\mathbf{x}, \mathbf{x}\rangle|}$. We denote by $\mathbf{x}^{\perp}$ the vector given by $\mathbf{x}^{\perp}=\left(x_{2}, x_{1}\right)$. Thus, $\mathbf{x}^{\perp}$ is orthogonal to $\mathbf{x}\left(\right.$ i.e.,$\left\langle\mathbf{x}, \mathbf{x}^{\perp}\right\rangle=0$ ) and $\|\mathbf{x}\|=\left\|\mathbf{x}^{\perp}\right\|$. We have $\mathbf{x}^{\perp}= \pm \mathbf{x}$ if and only if $\mathbf{x}$ is lightlike, and $\mathbf{x}^{\perp}$ is timelike (respectively, spacelike) if and only if $\mathbf{x}$ is spacelike (respectively, timelike).

Let $\zeta: I \longrightarrow \mathbb{R}_{1}^{2}$ be a spacelike or a timelike curve, we may take the arc-length parameter $s$ of $\zeta$. It follows that, $\left\|\zeta^{\prime}(s)\right\|=1$ for all $s \in I$, where $\zeta^{\prime}(s)=(d \zeta / d s)(s)$. We denote by $\mathbf{e}_{1}(s)$ the unit tangent vector and $\mathbf{e}_{2}(s)$ the unit normal vector to $\zeta(s)$ such that $\left\{\mathbf{e}_{1}(s), \mathbf{e}_{2}(s)\right\}$ is oriented anti-clockwise. Actually, $\mathbf{e}_{1}(s)=\zeta^{\prime}(s)$ and $\mathbf{e}_{2}(s)=(-1)^{w+1} \zeta^{\perp}$, where $w=1$ if $\zeta$ is a timelike and $w=2$ if $\zeta$ is a spacelike. So, we have the Serret-Frenet equations:

$$
\left(\begin{array}{l}
\mathbf{e}_{1}^{\prime}(s) \\
\mathbf{e}_{2}^{\prime}(s)
\end{array}\right)=\left(\begin{array}{cc}
0 & \kappa(s) \\
\kappa(s) & 0
\end{array}\right)\left(\begin{array}{l}
\mathbf{e}_{1}(s) \\
\mathbf{e}_{2}(s)
\end{array}\right),
$$

where $\kappa(s)$ is the curvature of $\zeta$ and is defined by

$$
\begin{aligned}
\kappa(s) & =\frac{\left\langle\mathbf{e}_{1}^{\prime}(s), \mathbf{e}_{2}(s)\right\rangle}{\left\langle\mathbf{e}_{2}(s), \mathbf{e}_{2}(s)\right\rangle} \\
& =(-1)^{w+1}\left\langle\mathbf{e}_{1}^{\prime}(s), \mathbf{e}_{2}(s)\right\rangle=\left\langle\zeta^{\prime \prime}(s), \zeta^{\prime \perp}\right\rangle .
\end{aligned}
$$

Even if $\zeta$ is not parameterized by the arc-length and $t$ denotes the parameter, then the unit tangent and the unit normal vectors of $\zeta(t)$ are oriented anti-clockwise and given by

$$
\mathbf{e}_{1}(t)=\frac{\dot{\zeta}(t)}{\|\dot{\zeta}(t)\|}, \quad \mathbf{e}_{2}(t)=(-1)^{w+1} \frac{\dot{\zeta}(t)^{\perp}}{\|\dot{\zeta}(t)\|} .
$$

Also, we have

$$
\left(\begin{array}{l}
\dot{\mathbf{e}_{1}}(t) \\
\dot{\mathbf{e}_{2}}(t)
\end{array}\right)=\left(\begin{array}{cc}
0 & \|\dot{\zeta}\| \kappa(t) \\
\|\dot{\zeta}\| \kappa(t) & 0
\end{array}\right)\left(\begin{array}{l}
\mathbf{e}_{1}(t) \\
\mathbf{e}_{2}(t)
\end{array}\right)
$$

and the curvature is given by

$$
\kappa(t)=\left\langle\ddot{\zeta}(t), \dot{\zeta}(t)^{\perp}\right\rangle /\|\dot{\zeta}(t)\|^{3},
$$

and we call a point $\zeta\left(t_{0}\right)$ an inflection point if $\left\langle\ddot{\zeta}(t), \dot{\zeta}(t)^{\perp}\right\rangle=0,14,15$.

An inflection point of a spacelike, or a timelike regular curve $\zeta$ is a point $\zeta(t)$ such that $\kappa(t)=$ 0 . Although at the arbitrary point selected, the definition of pedals is defined in $\mathbb{R}_{1}^{2}$, the origin is chosen. A pedal of $\zeta$ (with respect to $0 \in \mathbb{R}_{1}^{2}$ ) is defined to be $\operatorname{Pe}_{\zeta}(s)=\left\langle\zeta(s), \mathbf{e}_{2}(s)\right\rangle \mathbf{e}_{2}(s)$. At each point of the curve, the pedal of a curve is considered to be the locus of the projection image of the origin to the tangent line in the normal direction. Since

$$
\begin{aligned}
P e_{\zeta}^{\prime}(s)= & \kappa(s)\left(\left\langle\zeta(s), \mathbf{e}_{1}(s)\right\rangle \mathbf{e}_{2}(s)\right. \\
& \left.+\left\langle\zeta(s), \mathbf{e}_{2}(s)\right\rangle \mathbf{e}_{1}(s)\right)
\end{aligned}
$$

the singular points of the pedal of $\zeta$ are the point $s_{0}$ where $\zeta\left(s_{0}\right)=0$ or $\kappa\left(s_{0}\right)=0$ (i.e., $s_{0}$ is the inflection point of $\zeta$ ). If we take $\zeta$ does not pass through the origin, the singular points of the pedal $P e_{\zeta}$ are the inflection points of $\zeta$. The pedal curve is known to be the envelope of a family of circles, as follows:

Let $F: I \times \mathbb{R}_{1}^{2} \longrightarrow \mathbb{R}$ be a function defined by

$$
F(s, \mathbf{u})=\langle\mathbf{u}, \mathbf{u}-\zeta(s)\rangle
$$

we have the equation

$\partial F / \partial s(s, \mathbf{u})=\left\langle\mathbf{u},-\mathbf{e}_{1}(s)\right\rangle$. Since $\left\{\mathbf{e}_{1}(s), \mathbf{e}_{2}(s)\right\}$ is 
an orthonormal basis of $\mathbb{R}_{1}^{2}$, we can write $\mathbf{u}=\eta \mathbf{e}_{1}(s)+\xi \mathbf{e}_{2}(s)$ and we have

$$
\left\{\begin{array}{l}
F(s, \mathbf{u})=\partial F / \partial s(s, \mathbf{u})=0, \\
\langle\mathbf{u}, \mathbf{u}-\zeta(s)\rangle=\left\langle\mathbf{u},-\mathbf{e}_{1}(s)\right\rangle=0, \\
-\eta^{2}+\xi^{2}-\eta\left\langle\mathbf{e}_{1}(s), \zeta(s)\right\rangle-\xi\left\langle\mathbf{e}_{2}(s), \zeta(s)\right\rangle \\
=\eta=0
\end{array}\right.
$$

if and only if $\eta=0$ and $\xi\left(\xi-\left\langle\mathbf{e}_{2}(s), \zeta(s)\right\rangle\right)=0$. The final condition is equivalent to $\xi=0$ or $\mathbf{u}=\left\langle\zeta(s), \mathbf{e}_{2}(s)\right\rangle \mathbf{e}_{2}(s)$. This implies that the pedal $\mathrm{Pe}_{\zeta}(s)$ is the envelope of the above family of the circles. On the other hand, we define the evolute of $\zeta$ by

$$
E v_{\zeta}(s)=\zeta(s)-\frac{1}{\kappa(s)} \mathbf{e}_{2}(s),
$$

where $\kappa(s) \neq 0$. Evolute curve is known the osculating circle's center locus. And we get $E v_{\zeta}^{\prime}(s)=$ $\left(\kappa^{\prime 2}\right)(s) \mathbf{e}_{2}(s), s_{0} \in I$ is a singular point of $E v_{\zeta}$ if and only if $\kappa^{\prime}\left(s_{0}\right)=0$.

\section{Evolutoids and Pedaloids}

In this section, we are interested in the line $L$ obtained by rotating the tangent fixed angle $\varphi_{1}$. The direction of $L$ is therefore $\cos \varphi_{1} \mathbf{e}_{1}(s)+\sin \varphi_{1} \mathbf{e}_{2}(s)$ and $\sin \varphi_{1} \mathbf{e}_{1}(s)+\cos \varphi_{1} \mathbf{e}_{2}(s)$ is perpendicular to $L$. The vector equation of the line $L$ is $F(s, \mathbf{u})=$ 0 , where

$$
\begin{aligned}
F(s, \mathbf{u})= & (\mathbf{u}-\zeta(s))\left(\sin \varphi_{1} \mathbf{e}_{1}(s)+\cos \varphi_{1} \mathbf{e}_{2}(s)\right), \\
\frac{\partial F}{\partial s}(s, \mathbf{u})= & -\mathbf{e}_{1}(s)\left(\sin \varphi_{1} \mathbf{e}_{1}(s)+\cos \varphi_{1} \mathbf{e}_{2}(s)\right) \\
& +(\mathbf{u}-\zeta(s))\left(\kappa(s) \sin \varphi_{1} \mathbf{e}_{2}(s)\right. \\
& \left.+\kappa(s) \cos \varphi_{1} \mathbf{e}_{1}(s)\right) .
\end{aligned}
$$

Since any vector is a linear combination as $\eta \mathbf{e}_{1}(s)+\xi \mathbf{e}_{2}(s)$. So, for the vector $\mathbf{u}-\zeta(s)$ and by substituting in $F(s, \mathbf{u})=\frac{\partial F}{\partial s}(s, \mathbf{u})=0$, we obtain

$$
\left\{\begin{array}{l}
\left(\eta \mathbf{e}_{1}(s)+\xi \mathbf{e}_{2}(s)\right)\left(\sin \varphi_{1} \mathbf{e}_{1}(s)+\cos \varphi_{1} \mathbf{e}_{2}(s)\right)=0, \\
\sin \varphi_{1}+\left(\eta \mathbf{e}_{1}(s)+\xi \mathbf{e}_{2}(s)\right)\left(\kappa \sin \varphi_{1} \mathbf{e}_{2}(s)\right. \\
\left.+\kappa \cos \varphi_{1} \mathbf{e}_{1}(s)\right)=0,
\end{array}\right.
$$

it follows that

$$
\left\{\begin{array}{l}
-\eta \sin \varphi_{1}+\xi \cos \varphi_{1}=0, \\
-\eta \kappa \cos \varphi_{1}+\xi \kappa \sin \varphi_{1}=-\sin \varphi_{1} .
\end{array}\right.
$$

By solving these equations with respect to $\eta$ and $\xi$, we get

$$
\eta=\frac{-\sin \varphi_{1} \cos \varphi_{1}}{\kappa(s)\left(2 \sin ^{2} \varphi_{1}-1\right)}, \quad \xi=\frac{-\sin ^{2} \varphi_{1}}{\kappa(s)\left(2 \sin ^{2} \varphi_{1}-1\right)} .
$$

Since, $\mathbf{u}-\zeta(s)=\eta \mathbf{e}_{1}(s)+\xi \mathbf{e}_{2}(s)$, the concept of evolutoids is defined as follows:

For $\varphi_{1} \in[0,2 \pi)$, we have

$$
\begin{aligned}
E v\left[\varphi_{1}\right]_{\zeta}(s)= & \zeta(s)-\frac{\sin \varphi_{1}}{\kappa(s)\left(2 \sin ^{2} \varphi_{1}-1\right)}\left(\cos \varphi_{1} \mathbf{e}_{1}(s)\right. \\
& \left.+\sin \varphi_{1} \mathbf{e}_{2}(s)\right)
\end{aligned}
$$

where $\kappa(s) \neq 0$ and $\varphi_{1} \neq \pi / 4$. So, for each $\varphi_{1}$, Eq.(4) is called a $\varphi_{1}$-evolutoid of $\zeta$.

If $\varphi_{1}=\pi / 2,3 \pi / 2$, then $E v\left[\varphi_{1}\right]_{\zeta}(s)=E v_{\zeta}(s)$, and if $\varphi_{1}=0, \pi$, then $E v\left[\varphi_{1}\right]_{\zeta}(s)=\zeta(s)$.

By differentiation Eq. (4), we obtain

$$
\begin{aligned}
E v\left[\varphi_{1}\right]_{\zeta}^{\prime}= & \frac{-1}{\left(2 \sin ^{2} \varphi_{1}-1\right)}\left(\cos \varphi_{1}-\frac{\kappa^{\prime}}{\kappa^{2}} \sin \varphi_{1}\right) \\
& \left(\cos \varphi_{1} \mathbf{e}_{1}+\sin \varphi_{1} \mathbf{e}_{2}\right),
\end{aligned}
$$

and $s_{0} \in I$ is a singular point of $E v\left[\varphi_{1}\right]_{\zeta}$ if and only if $\kappa^{2}(s) \cos \varphi_{1}-\kappa^{\prime}(s) \sin \varphi_{1}=0$.

For $\varphi_{2} \in[0,2 \pi)$, we introduce the notion of pedaloids as follows:

$$
\begin{aligned}
\operatorname{Pe}\left[\varphi_{2}\right]_{\zeta}(s)= & \frac{1}{\left|2 \cos ^{2} \varphi_{2}-1\right|}\left\langle\zeta,-\cos \varphi_{2} \mathbf{e}_{1}+\sin \varphi_{2} \mathbf{e}_{2}\right\rangle \\
& \left(-\cos \varphi_{2} \mathbf{e}_{1}+\sin \varphi_{2} \mathbf{e}_{2}\right)
\end{aligned}
$$

we refer to this as $\varphi_{2}$-pedaloid of $\zeta$.

If $\varphi_{2}=\pi / 2,3 \pi / 2$, then $\operatorname{Pe}\left[\varphi_{2}\right]_{\zeta}(s)=P e_{\zeta}(s)$, and if $\varphi_{2}=0, \pi$, then $\operatorname{Pe}\left[\varphi_{2}\right]_{\zeta}(s)=\left\langle\zeta(s), \mathbf{e}_{1}(s)\right\rangle \mathbf{e}_{1}(s)$, this is classified as a counterpedal (i.e. a C-pedal for short), we refer to the C-pedal by $C P e_{\zeta}(s)$. By differentiation Eq. (6), we get

$$
\operatorname{Pe}\left[\varphi_{2}\right]_{\zeta}^{\prime}(s)=\frac{1}{\left|2 \cos ^{2} \varphi_{2}-1\right|}\left\{\begin{array}{l}
\left(-\cos ^{2} \varphi_{2}+\kappa\left\langle\zeta, \mathbf{e}_{2}\right\rangle\right. \\
\left.-\kappa \sin 2 \varphi_{2}\left\langle\zeta, \mathbf{e}_{1}\right\rangle\right) \mathbf{e}_{1} \\
+\left(\cos \varphi_{2} \sin \varphi_{2}\right. \\
+\kappa\left\langle\zeta, \mathbf{e}_{1}\right\rangle \\
\left.-\kappa \sin 2 \varphi_{2}\left\langle\zeta, \mathbf{e}_{2}\right\rangle\right) \mathbf{e}_{2}
\end{array}\right.
$$


It follows that

$$
\left\{\begin{array}{l}
\kappa(s)\left\langle\zeta(s), \mathbf{e}_{2}(s)\right\rangle \\
-\kappa(s) \sin 2 \varphi_{2}\left\langle\zeta(s), \mathbf{e}_{1}(s)\right\rangle=\cos ^{2} \varphi_{2}, \\
\kappa(s)\left\langle\zeta(s), \mathbf{e}_{1}(s)\right\rangle \\
-\kappa(s) \sin 2 \varphi_{2}\left\langle\zeta(s), \mathbf{e}_{2}(s)\right\rangle=-\cos \varphi_{2} \sin \varphi_{2},
\end{array}\right.
$$

for $\left\langle\zeta(s), \mathbf{e}_{2}(s)\right\rangle$ and $\left\langle\zeta(s), \mathbf{e}_{1}(s)\right\rangle$, hence $s_{0} \in I$ is a singular point of $\mathrm{Pe}\left[\varphi_{2}\right]_{\zeta}$ if and only if

$$
\begin{aligned}
& \kappa(s)\left\langle\zeta(s), \mathbf{e}_{2}(s)\right\rangle=\frac{\cos ^{2} \varphi_{2}\left(1-2 \sin ^{2} \varphi_{2}\right)}{1-4 \sin ^{2} \varphi_{2} \cos ^{2} \varphi_{2}}, \\
& \kappa(s)\left\langle\zeta(s), \mathbf{e}_{1}(s)\right\rangle=\Pi_{1} \sin \varphi_{2} \cos \varphi_{2},
\end{aligned}
$$

where

$$
\Pi_{1}=\left(1-\frac{2 \cos ^{2} \varphi_{2}\left(1-2 \sin ^{2} \varphi_{2}\right)}{1-4 \sin ^{2} \varphi_{2} \cos ^{2} \varphi_{2}}\right) .
$$

Thus, the relation between evolutoids and pedaloids is given in following theorem:

Theorem 1 Suppose that $\kappa(s) \neq 0, \varphi_{2} \neq \pi / 4$, and $\left(2 \sin ^{2} \varphi_{2}-1\right)\left(\kappa^{2} \cos \varphi_{2}-\kappa^{\prime} \sin \varphi_{2}\right) \neq 0$ (i.e., the $\varphi_{2}+\pi / 2$-evolutoid is non-singular). Then, the following equation is satisfied

$$
P e_{E v}\left[\varphi_{2}+\pi / 2\right]_{\zeta}(s)=\operatorname{Pe}\left[\varphi_{2}\right]_{\zeta}(s)
$$

Proof: Since $F\left[\varphi_{2}\right]: I \times \mathbb{R}_{1}^{2} \longrightarrow \mathbb{R}$, where

$$
F\left[\varphi_{2}\right](s, \mathbf{u})=\left\langle\mathbf{u}, \mathbf{u}-E v\left[\varphi_{2}+\pi / 2\right]_{\zeta}(s)\right\rangle .
$$

Because $E v\left[\varphi_{2}+\pi / 2\right]_{\zeta}(s)$ is a regular curve, so the envelope is given by $F\left[\varphi_{2}\right](s, \mathbf{u})=0$, and it is the pedal $P e_{E v}\left[\varphi_{2}+\pi / 2\right]_{\zeta}(s)$ of $E v\left[\varphi_{2}+\pi / 2\right]_{\zeta}(s)$.

From Eq. (4), we get

$$
E v\left[\varphi_{1}\right]_{\zeta}^{\prime}(s)=\Pi_{2}\left(\cos \varphi_{1} \mathbf{e}_{1}(s)+\sin \varphi_{1} \mathbf{e}_{2}(s)\right),
$$

where

$$
\Pi_{2}=\frac{-1}{\left(2 \sin ^{2} \varphi_{1}-1\right)}\left(\cos \varphi_{1}-\frac{\kappa^{\prime}}{\kappa^{2}} \sin \varphi_{1}\right) .
$$

Also, we obtain

$$
\frac{\partial F\left[\varphi_{2}\right]}{\partial s}(s, \mathbf{u})=\Pi_{2}\left\langle\mathbf{u}, \cos \varphi_{1} \mathbf{e}_{1}(s)+\sin \varphi_{1} \mathbf{e}_{2}(s)\right\rangle .
$$

Since $\left\{\mathbf{e}_{1}, \mathbf{e}_{2}\right\}$ is an orthonormal frame, there exist $\eta, \xi \in \mathbb{R}$, such that $\mathbf{u}=\eta \mathbf{e}_{1}(s)+\xi \mathbf{e}_{2}(s)$, it follows that

$$
\frac{\partial F\left[\varphi_{2}\right]}{\partial s}(s, \mathbf{u})=\Pi_{2}\left\langle\eta \mathbf{e}_{1}+\xi \mathbf{e}_{2}, \cos \varphi_{1} \mathbf{e}_{1}+\sin \varphi_{1} \mathbf{e}_{2}\right\rangle,
$$

where $\varphi_{1}=\varphi_{2}+\pi / 2$.

From which, $\frac{\partial F\left[\varphi_{2}\right]}{\partial s}(s, \mathbf{u})=0$ if and only if $-\eta \cos \left(\varphi_{2}+\pi / 2\right)+\xi \sin \left(\varphi_{2}+\pi / 2\right)=0$, which is equivalent to $-\eta \sin \varphi_{2}-\xi \cos \varphi_{2}=0$.

For $\varphi_{1}=\varphi_{2}+\pi / 2$, we have

$$
\begin{aligned}
F\left[\varphi_{1}\right](s, \mathbf{u})= & \left\langle\mathbf{u}, \mathbf{u}-E v\left[\varphi_{1}\right]_{\zeta}(s)\right\rangle, \\
= & -\eta^{2}+\xi^{2}-\eta\left\langle\mathbf{e}_{1}, \zeta\right\rangle-\xi\left\langle\mathbf{e}_{2}, \zeta\right\rangle \\
& +\frac{\sin \varphi_{1}}{\kappa\left(2 \sin ^{2} \varphi_{1}-1\right)}\left(-\eta \cos \varphi_{1}\right. \\
& \left.+\xi \sin \varphi_{1}\right), \\
= & -\eta^{2}+\xi^{2}-\eta\left\langle\mathbf{e}_{1}, \zeta\right\rangle-\xi\left\langle\mathbf{e}_{2}, \zeta\right\rangle .
\end{aligned}
$$

Let $\varphi_{2} \neq 0, \pi$ (i.e., $\sin \varphi_{2}=\cos \varphi_{1} \neq 0$ ) and using $\frac{\partial F\left[\varphi_{2}\right]}{\partial s}(s, \mathbf{u})=0$, we obtain

$$
\eta=\xi \frac{\sin \varphi_{1}}{\cos \varphi_{1}} .
$$

From Eq. (8), we get

$$
-\xi^{2} \frac{\sin ^{2} \varphi_{1}}{\cos ^{2} \varphi_{1}}+\xi^{2}-\xi \frac{\sin \varphi_{1}}{\cos \varphi_{1}}\left\langle\mathbf{e}_{1}, \zeta\right\rangle-\xi\left\langle\mathbf{e}_{2}, \zeta\right\rangle=0,
$$

it follows that, $\eta=\xi=0$ or $\xi \neq 0$. If $\xi \neq 0$, then

$$
\begin{aligned}
\xi & =\frac{-\cos \varphi_{1}}{2 \sin ^{2} \varphi_{1}-1}\left(\sin \varphi_{1}\left\langle\mathbf{e}_{1}, \zeta\right\rangle+\cos \varphi_{1}\left\langle\mathbf{e}_{2}, \zeta\right\rangle\right), \\
\eta & =\frac{-\sin \varphi_{1}}{2 \sin ^{2} \varphi_{1}-1}\left(\sin \varphi_{1}\left\langle\mathbf{e}_{1}, \zeta\right\rangle+\cos \varphi_{1}\left\langle\mathbf{e}_{2}, \zeta\right\rangle\right),
\end{aligned}
$$

and then, we obtain

$$
\begin{aligned}
\mathbf{u}= & \eta \mathbf{e}_{1}(s)+\xi \mathbf{e}_{2}(s) \\
= & \frac{-\sin \varphi_{1}}{2 \sin ^{2} \varphi_{1}-1}\left(\sin \varphi_{1}\left\langle\mathbf{e}_{1}, \zeta\right\rangle+\cos \varphi_{1}\left\langle\mathbf{e}_{2}, \zeta\right\rangle\right) \mathbf{e}_{1} \\
& -\frac{\cos \varphi_{1}}{2 \sin ^{2} \varphi_{1}-1}\left(\sin \varphi_{1}\left\langle\mathbf{e}_{1}, \zeta\right\rangle+\cos \varphi_{1}\left\langle\mathbf{e}_{2}, \zeta\right\rangle\right) \mathbf{e}_{2} \\
= & \frac{-1}{2 \cos ^{2} \varphi_{2}-1}\left(\left\langle-\cos \varphi_{2} \mathbf{e}_{1}+\sin \varphi_{2} \mathbf{e}_{2}, \zeta\right\rangle\right) \\
& \left(\cos \varphi_{2} \mathbf{e}_{1}-\sin \varphi_{2} \mathbf{e}_{2}\right) \\
= & \operatorname{Pe}\left[\varphi_{2}\right]_{\zeta}(s) .
\end{aligned}
$$


If $\eta=\xi=0$, then $\mathbf{u}=0$ satisfies the condition $F\left[\varphi_{1}\right]\left(s_{0}, \mathbf{u}\right)=\frac{\partial F\left[\varphi_{1}\right]}{\partial s}\left(s_{0}, \mathbf{u}\right)=0$, it follows that

$$
\begin{aligned}
& \left\langle\sin \varphi_{1} \mathbf{e}_{1}\left(s_{0}\right)+\cos \varphi_{1} \mathbf{e}_{2}\left(s_{0}\right), \zeta\left(s_{0}\right)\right\rangle \\
& =\sin \varphi_{1}\left\langle\mathbf{e}_{1}\left(s_{0}\right), \zeta\left(s_{0}\right)\right\rangle+\cos \varphi_{1}\left\langle\mathbf{e}_{2}\left(s_{0}\right), \zeta\left(s_{0}\right)\right\rangle=0 .
\end{aligned}
$$

Thus, $\operatorname{Pe}\left[\varphi_{2}\right]_{\zeta}\left(s_{0}\right)=\mathbf{u}=0$ and it means that, the envelope of the family $F\left[\varphi_{2}\right](s, \mathbf{u})=0$ is the $\varphi_{2}$-pedaloid $\mathrm{Pe}\left[\varphi_{2}\right]_{\zeta}(s)$. Hence, the proof is completed.

\section{Pedaloids and Evolutoids of Frontals}

The notion of frontals is one of the natural singular curves, so we can establish the differential geometry of these curves in the Minkowski plane. We say that $(\zeta, \boldsymbol{\nu}): I \longrightarrow \mathbb{R}_{1}^{2} \times S_{1}^{1}$ is a Legendrian curve if $(\zeta, \boldsymbol{\nu})^{*} \theta=0$, where $\theta$ is the canonical contact 1 -form on the unit tangent bundle $\mathbf{e}_{1} \mathbb{R}_{1}^{2}=\mathbb{R}_{1}^{2} \times S_{1}^{1}$.

Furthermore, $(\zeta, \boldsymbol{\nu})^{*} \theta=0$ is equivalent to $\langle\dot{\zeta}(t), \boldsymbol{\nu}(t)\rangle=0$ for any $t \in I$.

So, $\zeta: I \longrightarrow \mathbb{R}_{1}^{2}$ is a frontal if there exists $\boldsymbol{\nu}: I \longrightarrow S_{1}^{1}$ such that $(\zeta, \boldsymbol{\nu})$ is a Legendrian curve. If $(\zeta, \boldsymbol{\nu})$ is an immersion, $\zeta$ is said to be a front. A differential geometry on frontals was constructed in [4]. For a Legendrian curve $(\zeta, \boldsymbol{\nu}): I \longrightarrow \mathbb{R}_{1}^{2} \times S_{1}^{1}$, we define a unit vector field $\boldsymbol{\mu}(t)=J(\boldsymbol{\nu}(t))$ along $\zeta$.

Then, we have the following Serret-Frenet equations:

$$
\left(\begin{array}{l}
\dot{\boldsymbol{\nu}}(t) \\
\dot{\boldsymbol{\mu}}(t)
\end{array}\right)=\left(\begin{array}{cc}
0 & \ell(t) \\
\ell(t) & 0
\end{array}\right)\left(\begin{array}{l}
\boldsymbol{\nu}(t) \\
\boldsymbol{\mu}(t)
\end{array}\right),
$$

where $\ell(t)=\langle\dot{\boldsymbol{\nu}}(t), \boldsymbol{\mu}(t)\rangle$. Moreover, there exists $\beta(t)$ such that $\dot{\zeta}(t)=\beta(t) \boldsymbol{\mu}(t)$ for any $t \in I$. The pair $(\ell, \beta)$ is called a curvature of the Legendrian curve $(\zeta, \boldsymbol{\nu})$. By definition, $t_{0} \in I$ is a singular point of $\zeta$ if and only if $\beta\left(t_{0}\right)=0$. Furthermore, for a regular curve $\zeta$, we have $\boldsymbol{\mu}(t)=\mathbf{e}_{1}(t)$ and $\ell(t)=\|\dot{\zeta}(t)\| \kappa(t)$. The Legendrian curve $(\zeta, \boldsymbol{\nu})$ is immersive (i.e., $\zeta$ is a front) if and only if $(\ell(t), \beta(t)) \neq(0,0)$ for any $t \in I$. So, the inflection point $t_{0} \in I$ of the frontal $\zeta$ is a point $\ell\left(t_{0}\right)=0$ (for more details, see [8, 15,17$\left.]\right)$.

The evolute of a frontal $\zeta$ is defined by

$$
\mathcal{E} v_{\zeta}(t)=\zeta(t)-\frac{\beta(t)}{\ell(t)} \boldsymbol{\nu}(t)
$$

by using the hypothesis that there exists $\alpha(t)$ such that $\beta(t)=\alpha(t) \ell(t)$ for any $t \in I$. Further, $\mathcal{E} v_{\zeta}(t)$ is a frontal and it is a front if $\zeta$ is a front 9 .

For line $L^{*}$ obtained by rotating the tangent fixed angle $\varphi_{1}$; the direction of $L^{*}$ is $\cos \varphi_{1} \boldsymbol{\mu}(t)+$ $\sin \varphi_{1} \boldsymbol{\nu}(t)$. Because, $\sin \varphi_{1} \boldsymbol{\mu}(t)+\cos \varphi_{1} \boldsymbol{\nu}(t)$ is perpendicular to $L^{*}$, the vector equation of the line $L^{*}$ is given by $G(t, \mathbf{x})=0$, where

$$
\begin{aligned}
& G(t, \mathbf{x})=(\mathbf{x}-\zeta(t))\left(\sin \varphi_{1} \boldsymbol{\mu}(t)+\cos \varphi_{1} \boldsymbol{\nu}(t)\right), \\
& \frac{\partial G}{\partial t}(t, \mathbf{x})=-\beta(t) \boldsymbol{\mu}(t)\left(\sin \varphi_{1} \boldsymbol{\mu}(t)+\cos \varphi_{1} \boldsymbol{\nu}(t)\right)+ \\
&(\mathbf{x}-\zeta(t))\left(\ell(t) \sin \varphi_{1} \boldsymbol{\nu}(t)+\ell(t) \cos \varphi_{1} \boldsymbol{\mu}(t)\right) .
\end{aligned}
$$

From which, any vector is a linear combination in the form $\eta_{1} \boldsymbol{\mu}(t)+\eta_{2} \boldsymbol{\nu}(t)$. Using this to the vector $\mathbf{x}-\zeta(t)$ and substituting in $G(t, \mathbf{x})=\frac{\partial G}{\partial t}(t, \mathbf{x})=$ 0 , we obtain

$$
\left\{\begin{array}{l}
\left(\eta_{1} \boldsymbol{\mu}(t)+\eta_{2} \boldsymbol{\nu}(t)\right)\left(\sin \varphi_{1} \boldsymbol{\mu}(t)+\cos \varphi_{1} \boldsymbol{\nu}(t)\right)=0, \\
\beta(t) \sin \varphi_{1}+\left(\eta_{1} \boldsymbol{\mu}(t)+\eta_{2} \boldsymbol{\nu}(t)\right)\left(\ell(t) \sin \varphi_{1} \boldsymbol{\nu}(t)\right. \\
\left.+\ell(t) \cos \varphi_{1} \boldsymbol{\mu}(t)\right)=0,
\end{array}\right.
$$

it follows that

$$
\left\{\begin{array}{l}
-\eta_{1} \sin \varphi_{1}+\eta_{2} \cos \varphi_{1}=0, \\
-\eta_{1} \ell(t) \cos \varphi_{1}+\eta_{2} \ell(t) \sin \varphi_{1}=-\beta(t) \sin \varphi_{1} .
\end{array}\right.
$$

By solving these equations with respect to $\eta_{1}$ and $\eta_{2}$, we get

$\eta_{1}=\frac{-\beta(t) \sin \varphi_{1} \cos \varphi_{1}}{\ell(t)\left(2 \sin ^{2} \varphi_{1}-1\right)}, \quad \eta_{2}=\frac{-\beta(t) \sin ^{2} \varphi_{1}}{\ell(t)\left(2 \sin ^{2} \varphi_{1}-1\right)}$.

Since, $\mathbf{x}-\zeta(t)=\eta_{1} \boldsymbol{\mu}(t)+\eta_{2} \boldsymbol{\mu}(t)$, the notion of evolutoids is defined by

$$
\begin{aligned}
\mathcal{E} v_{\zeta}\left[\varphi_{1}\right](t)= & \zeta-\frac{\beta}{\ell} \frac{\sin \varphi_{1}}{\left(2 \sin ^{2} \varphi_{1}-1\right)}\left(\cos \varphi_{1} \boldsymbol{\mu}\right. \\
& \left.+\sin \varphi_{1} \boldsymbol{\nu}\right)
\end{aligned}
$$

it leads to $\mathcal{E} v_{\zeta}[0](t)=\mathcal{E} v_{\zeta}[\pi](t)=\zeta(t)$ and $\mathcal{E} v_{\zeta}[\pi / 2](t)=\mathcal{E} v_{\zeta}[3 \pi / 2](t)=\mathcal{E} v_{\zeta}(t)$.

Proposition 2 Let $(\boldsymbol{\zeta}, \boldsymbol{\nu})$ be a Legendrian curve with the curvature $(\ell, \beta)$, and there exists $\alpha(t)$ where $\beta(t)=\alpha(t) \ell(t)$ for any $t \in I$. Then, the $\varphi_{1}$-evolutoid $\mathcal{E} v_{\zeta}\left[\varphi_{1}\right]$ is a frontal with the curvature $(\ell, \ell)$. If $\ell(t) \neq 0$, then $\mathcal{E} v_{\zeta}\left[\varphi_{1}\right]$ is a front. 
Proof: By differentiation Eq. (11), we get

$$
\begin{aligned}
\frac{d}{d t} \mathcal{E} v_{\zeta}\left[\varphi_{1}\right](t)= & -\frac{1}{2 \sin ^{2} \varphi_{1}-1}\left(\beta(t) \cos \varphi_{1}\right. \\
& \left.+\dot{\alpha}(t) \sin \varphi_{1}\right)\left(\cos \varphi_{1} \boldsymbol{\mu}(t)\right. \\
& \left.+\sin \varphi_{1} \boldsymbol{\nu}(t)\right)
\end{aligned}
$$

And then, we obtain

$$
\begin{aligned}
\boldsymbol{\nu}\left[\varphi_{1}\right](t) & =J\left(\cos \varphi_{1} \boldsymbol{\mu}(t)+\sin \varphi_{1} \boldsymbol{\nu}(t)\right) \\
& =\left(\sin \varphi_{1} \boldsymbol{\mu}(t)+\cos \varphi_{1} \boldsymbol{\nu}(t)\right)
\end{aligned}
$$

If $\left(\mathcal{E} v_{\zeta}\left[\varphi_{1}\right], \nu\left[\varphi_{1}\right]\right): I \longrightarrow \mathbb{R}_{1}^{2} \times S_{1}^{1}$, then

$$
\left\langle\frac{d}{d t} \mathcal{E} v_{\zeta}\left[\varphi_{1}\right](t), \boldsymbol{\nu}\left[\varphi_{1}\right](t)\right\rangle=0
$$

it follows that $\left(\mathcal{E} v_{\zeta}\left[\varphi_{1}\right], \boldsymbol{\nu}\left[\varphi_{1}\right]\right)$ is a Legendrian curve, which means that $\mathcal{E} v_{\zeta}\left[\varphi_{1}\right]$ is a frontal.

Furthermore, we obtain

$\boldsymbol{\mu}\left[\varphi_{1}\right](t)=J \boldsymbol{\nu}\left[\varphi_{1}\right](t)=-\left(\cos \varphi_{1} \boldsymbol{\mu}(t)+\sin \varphi_{1} \boldsymbol{\nu}(t)\right)$,

and

$$
\frac{d}{d t} \boldsymbol{\nu}\left[\varphi_{1}\right](t)=\ell(t)\left(\cos \varphi_{1} \boldsymbol{\mu}(t)+\sin \varphi_{1} \boldsymbol{\nu}(t)\right),
$$

where

$$
\begin{aligned}
\ell\left[\varphi_{1}\right](t) & =\left\langle\frac{d}{d t} \boldsymbol{\mu}\left[\varphi_{1}\right](t), \boldsymbol{\nu}\left[\varphi_{1}\right](t)\right\rangle=\ell(t), \\
\frac{d}{d t} \boldsymbol{\nu}\left[\varphi_{1}\right](t) & =-\ell(t) \boldsymbol{\mu}\left[\varphi_{1}\right](t) .
\end{aligned}
$$

Then, the curvature of $\mathcal{E} v_{\zeta}\left[\varphi_{1}\right]$ is $(\ell, \ell)$, and if $\ell(t) \neq 0$, then $\left(\mathcal{E} v_{\zeta}\left[\varphi_{1}\right], \boldsymbol{\nu}\left[\varphi_{1}\right]\right)$ is immersive. Hence, this completes the proof.

In what follows, we define a $\varphi_{2}$-pedaloid of a frontal $\zeta$ as:

$$
\begin{aligned}
\mathcal{P} e\left[\varphi_{2}\right]_{\zeta}(t)= & \frac{1}{\left|2 \cos ^{2} \varphi_{2}-1\right|}\left\langle\zeta,-\cos \varphi_{2} \boldsymbol{\mu}+\sin \varphi_{2} \boldsymbol{\nu}\right\rangle \\
& \left(-\cos \varphi_{2} \boldsymbol{\mu}(t)+\sin \varphi_{2} \boldsymbol{\nu}(t)\right)
\end{aligned}
$$

For a Legendrian curve $(\zeta, \boldsymbol{\nu})$, we are able to define the $\varphi_{2}$ as follows

$$
\begin{aligned}
\mathcal{P} e_{\zeta}(t) & =\mathcal{P} e[\pi / 2]_{\zeta}(t)=\mathcal{P} e[3 \pi / 2]_{\zeta}(t), \\
\mathcal{C} \mathcal{P} e_{\zeta}(t) & =\mathcal{P} e[0]_{\zeta}(t)=\mathcal{P} e[\pi]_{\zeta}(t) .
\end{aligned}
$$

We call $\mathcal{P} e_{\zeta}(t)$ as a pedal of $\zeta$ and $\mathcal{C} \mathcal{P} e_{\zeta}(t)$ a contrapedal of $\zeta$ 17, 18.
Proposition 3 By using a hypothesis that there exist $\delta(t)$ and $\boldsymbol{\sigma}: I \longrightarrow S_{1}^{1}$ such that $\zeta(t)=$ $\delta(t) \boldsymbol{\sigma}(t)$ for any $t \in I$. Let $(\zeta, \boldsymbol{\nu})$ be a Legendrian curve with the curvature $(\ell, \beta)$, then the pedal $\mathcal{P} e_{\zeta}(t)$ of $\zeta$ is a frontal.

Proof: By differentiation Eq. ([2), we get

$$
\begin{aligned}
\frac{d}{d t} \mathcal{P} e_{\zeta}(t) & =\ell(t)(\langle\zeta(t), \boldsymbol{\nu}(t)\rangle \boldsymbol{\mu}(t)+\langle\zeta(t), \boldsymbol{\mu}(t)\rangle \boldsymbol{\nu}(t)), \\
& =\ell(t) \delta(t)(\langle\boldsymbol{\sigma}, \boldsymbol{\nu}\rangle \boldsymbol{\mu}(t)+\langle\boldsymbol{\sigma}, \boldsymbol{\mu}\rangle \boldsymbol{\nu}(t)) .
\end{aligned}
$$

The unit vector field $\overline{\boldsymbol{\nu}}$ along $\zeta$ can be defined as

$$
\begin{aligned}
\overline{\boldsymbol{\nu}}(t)= & \frac{1}{\sqrt{\left|\langle\boldsymbol{\sigma}, \boldsymbol{\mu}\rangle^{2}+\langle\boldsymbol{\sigma}, \boldsymbol{\nu}\rangle^{2}\right|}}(\langle\boldsymbol{\sigma}(t), \boldsymbol{\mu}(t)\rangle \boldsymbol{\mu}(t) \\
& +\langle\boldsymbol{\sigma}(t), \boldsymbol{\nu}(t)\rangle \boldsymbol{\nu}(t)),
\end{aligned}
$$

it follows that $\left\langle\frac{d}{d t} \mathcal{P} e_{\zeta}(t), \bar{\nu}(t)\right\rangle=0$. Thus, the proof is completed.

From Proposition 3, we note that it is satisfied if $\zeta(t)=0$. So, the curvature of $\mathcal{P} e_{\zeta}(t)$ is rather complicated even for regular curve $\zeta$. Then, we obtain the next generalization of Theorem 1 .

Theorem 4 Let $(\zeta, \nu)$ be a Legendrian curve with the curvature $(\ell, \beta)$, and there exists $\alpha(t)$ such that $\beta(t)=\alpha(t) \ell(t)$ for any $t \in I$. Then we have

$$
\mathcal{P} e\left[\varphi_{2}\right]_{\zeta}(t)=\mathcal{P} e_{\mathcal{E} v_{\zeta}\left[\varphi_{2}+\pi / 2\right]}(t) .
$$

Proof: From aforementioned calculations, we have

$$
\begin{aligned}
\mathcal{P} e_{\mathcal{E} v_{\zeta}\left[\varphi_{1}\right]}(t)= & \left\langle\mathcal{E} v_{\zeta}\left[\varphi_{1}\right](t), \boldsymbol{\nu}\left[\varphi_{1}\right](t)\right\rangle \boldsymbol{\nu}\left[\varphi_{1}\right](t) \\
= & \left\langle\zeta, \sin \varphi_{1} \boldsymbol{\mu}+\cos \varphi_{1} \boldsymbol{\nu}\right\rangle\left(\sin \varphi_{1} \boldsymbol{\mu}\right. \\
& \left.+\cos \varphi_{1} \boldsymbol{\nu}\right)
\end{aligned}
$$

Using $\varphi_{2}+\pi / 2$ instead of $\varphi_{1}$, we get

$$
\begin{aligned}
\mathcal{P} e_{\mathcal{E} v_{\zeta}\left[\varphi_{2}+\pi / 2\right]}(t)= & \left\langle\zeta(t),-\cos \varphi_{2} \boldsymbol{\mu}(t)+\sin \varphi_{2} \boldsymbol{\nu}(t)\right\rangle \\
& \left(-\cos \varphi_{2} \boldsymbol{\mu}(t)+\cos \varphi_{2} \boldsymbol{\nu}(t)\right), \\
& =\mathcal{P} e\left[\varphi_{2}\right]_{\zeta}(t) .
\end{aligned}
$$

Hence, this completes the proof.

Corollary $\mathbf{5}$ Let $(\zeta, \boldsymbol{\nu})$ be a Legendrian curve with the curvatue $(\ell, \beta)$, and there exists $\alpha(t), \delta(t)$ and $\boldsymbol{\sigma}: I \longrightarrow S_{1}^{1}$ such that $\beta(t)=\alpha(t) \ell(t)$ and $\mathcal{E} v_{\zeta}\left[\varphi_{2}+\pi / 2\right](t)=\delta(t) \boldsymbol{\sigma}(t)$ for any $t \in I$. Then the $\varphi_{2}$-pedaloid $\mathcal{P} e\left[\varphi_{2}\right]_{\zeta}$ is a frontal. 


\section{Computational Examples}

In this section, we give and illustrate two computational examples to investigate some geometric properties of the evolutoid and pedaloid curves in Minkowski plane.

Example 5.1 Consider the curve

$\zeta_{1}:[-\pi, \pi] \longrightarrow \mathbb{R}_{1}^{2}$ defined by

$$
\zeta_{1}(t)=\frac{1}{2}\left(\cos ^{3}(t), \sin ^{3}(t)\right)
$$

then, we get

$$
\left\{\begin{array}{l}
\boldsymbol{\nu}(t)=\frac{1}{\rho_{1}}(-\sin (t), \cos (t)), \\
\boldsymbol{\mu}(t)=\frac{1}{\rho_{1}}(-\cos (t), \sin (t)), \\
\ell(t)=\frac{-1}{\rho_{1}^{2}}, \quad \beta(t)=\frac{3}{2} \rho \cos (t) \sin (t), \\
\alpha(t)=-\frac{3}{2} \rho_{1}^{3} \cos (t) \sin (t),
\end{array}\right.
$$

where $\rho_{1}(t)=\sqrt{\left|\sin ^{2}(t)-\cos ^{2}(t)\right|}$, therefore we obtain $\left\langle\dot{\zeta}_{1}(t), \boldsymbol{\nu}(t)\right\rangle=0$ and $(\ell(t), \beta(t)) \neq(0,0)$. Hence, we can easily show that $\zeta_{1}$ is a front. The curve $\zeta_{1}$ and 0 -pedaloid $\mathcal{P} e[0]_{\zeta_{1}}$ are shown in Figs. $1 a$ and $1 b$, respectively.

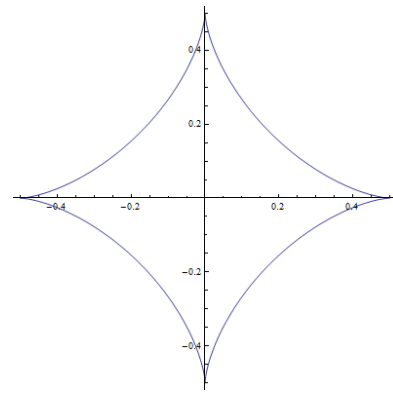

(a)

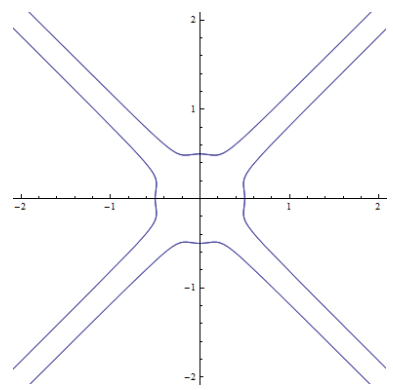

(b)
Figure 1: $(a) \zeta_{1}(t)=\frac{1}{2}\left(\cos ^{3}(t), \sin ^{3}(t)\right)$.

(b) The 0 -pedaloid of $\zeta_{1}$.

Also, the $\pi / 2$-pedaloid $\mathcal{P} e[\pi / 2]_{\zeta_{1}}$ is shown in Fig. 2a and the pedaloids altogether are shown in Fig. 2b.

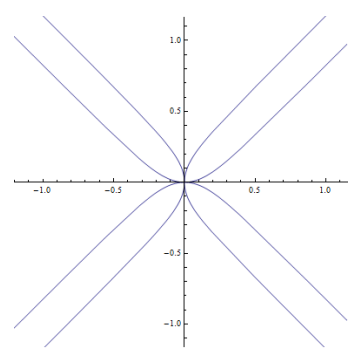

(a)

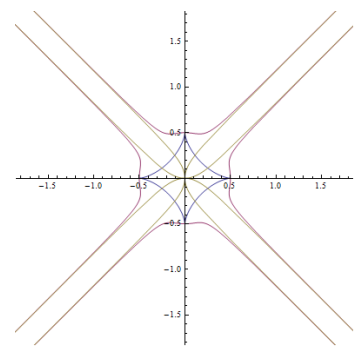

(b)
Figure 2: (a) The $\pi / 2$-pedaloid of $\zeta_{1}$. (b) $\zeta_{1}$ and pedaloids.

On the other hand, the $\pi / 2$-evolutoid $\mathcal{E} v_{\zeta_{1}}[\pi / 2]$ and 0 -evolutoid $\mathcal{E} v_{\zeta_{1}}[0]$ are shown in Figs. [3a and $3 b$, respectively.

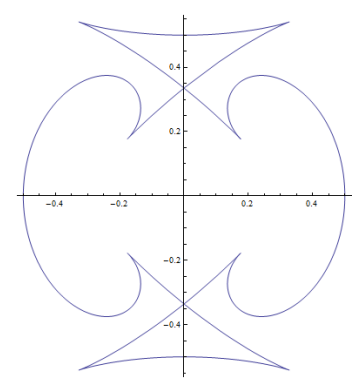

(a)

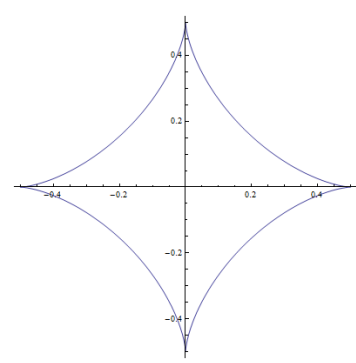

(b)
Figure 3: (a) The $\pi / 2$-evolutoid of $\zeta_{1}$. (b) The 0 -evolutoid of $\zeta_{1}$.

Furthermore, the evolutoids altogether are shown in Fig. 4.

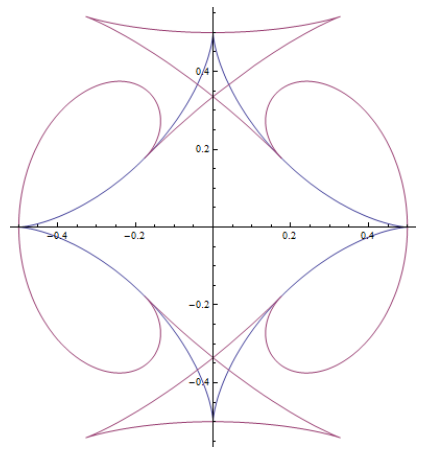

Figure 4: Evolutoids of $\zeta_{1}$. 
Example 5.2 Consider $\zeta_{2}:[-\pi, \pi] \longrightarrow \mathbb{R}_{1}^{2}$ is given by

$$
\zeta_{2}(t)=(\cos (t), \cos (t) \sin (t)),
$$

then, we obtain

$$
\left\{\begin{array}{l}
\boldsymbol{\nu}(t)=\frac{1}{\rho_{2}}(-\cos (2 t), \sin (t)), \\
\boldsymbol{\mu}(t)=\frac{1}{\rho_{2}}(-\sin (t), \cos (2 t)), \\
\ell(t)=\frac{1}{\rho_{2}^{2}}(-2 \sin (t) \sin (2 t)+\cos (t) \cos (2 t)), \\
\beta(t)=\rho_{2}(t), \\
\alpha(t)=\frac{1}{\rho_{2}}(-2 \sin (t) \sin (2 t)+\cos (t) \cos (2 t)),
\end{array}\right.
$$

where $\rho_{2}(t)=\sqrt{\left|\sin ^{2}(t)-\cos ^{2}(2 t)\right|}$, then we have $\left\langle\dot{\zeta}_{2}(t), \boldsymbol{\nu}(t)\right\rangle=0$ and $(\ell(t), \beta(t)) \neq(0,0)$. Therefore, we can easily show that $\zeta_{2}$ is a front. The curve $\zeta_{2}$ is shown in Fig. $5 a$ and the 0-pedaloid $\mathcal{P} e[0]_{\zeta_{2}}$ is shown in Fig. $5 b$.

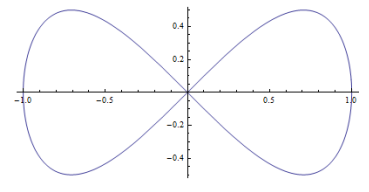

(a)

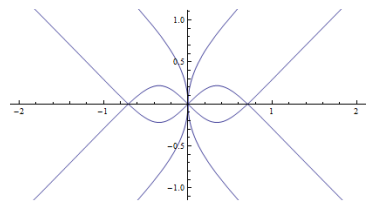

(b)
Figure 5: $(a) \zeta_{2}(t)=(\cos (t), \cos (t) \sin (t))$.

(b) The 0-pedaloid of $\zeta_{2}$.

Also, the $\pi / 2$-pedaloid $\mathcal{P} e[\pi / 2]_{\zeta_{2}}$ is shown in Fig. 6a, and we obtain the pedaloids altogether as shown in Fig. 6b.

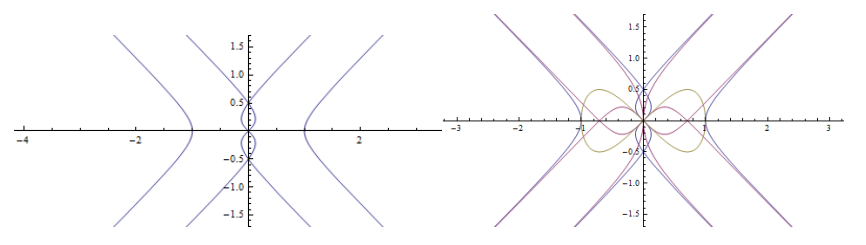

(a)

(b)

Figure 6: $(a)$ The $\pi / 2$-pedaloid of $\zeta_{2}$.

(b) $\zeta_{2}$ and pedaloids.

After that, we can get the images of $\pi / 2$ evolutoid $\mathcal{E} v_{\zeta_{2}}[\pi / 2]$ and 0 -evolutoid $\mathcal{E}_{\zeta_{2}}[0]$ as shown in Figs. $7 a$ and $7 \mathrm{~b}$, respectively.

Finally, we obtain the images of evolutoids altogether, as shown in Fig. 8.

E-ISSN: 2224-2880 


\section{References}

[1] F. Catoni, et al., The mathematics of Minkowski space-time, Birkhäuser Verlag $A G, 2008$.

[2] F. Catoni, et al., Geometry of Minkowski space-time, Springer Science $\mathscr{6}$ Business Media, 2011.

[3] G. Naber, The geometry of Minkowski spacetime: An introduction to the mathematics of the special theory of relativity, second ed., Springer Science \& Business Media, 2012.

[4] G. Naber, Spacetime and singularities, Cambridge University Press, Cambridge, England, 1988.

[5] G. Naber, Topology, Geometry and Gauge fields: Interactions, second ed., Springer, New York, 2011.

[6] T. Nishimura, Normal forms for singularities of pedal curves produced by non-singular dual curve germs in $S^{n}$, Geometriae Dedicata 133, 2008, pp. 59-66.

[7] M. Božek and G. Foltán, On singularities of arbitrary order of pedal curves, Proc. Symp. Comput. Geom. (SCG) 21, 2012, pp. 22-27.

[8] T. Fukunaga and M. Takahashi, Evolutes and involutes of frontals in the Euclidean plane, Demonstratio Mathematica XLVIII, 2015, pp. 147-166.

[9] T. Fukunaga, M. Takahashi, Existence and uniqueness for Legendre curves, J. Geom. 104, 2013, pp. 297-307.

[10] T. Fukunaga, M. Takahashi, Evolutes of fronts in the Euclidean plane, J. Singul. 10, 2014, pp. 92-107.

[11] V. I. Arnold, Singularities of caustics and wave fronts, Kluwer Academic Publishers, Dordrecht, 1990.

[12] Y. Li and D. Pei, Pedal curves of fronts in the sphere, J. Nonlinear Sci. Appl. 9, 2016, pp. 836-844.

[13] P. J. Giblin and J. P. Warder, Evolving evolutoids, American Mathematical Monthly 121, 2014, pp. 871-889.

[14] J. W. Bruce, P. J. Giblin, Curves and singularities, A geometrical introduction to singularity theory, Cambridge University Press, Cambridge, 1992.

[15] Y. Li, Q. Sun, Evolutes of fronts in the Minkowski plane, Math. Meth. Appl. Sci. 42, 2018, pp. 1-11.

[16] Y. Haiou, P. Donghe and C. Xiupeng, Evolutes of fronts on Euclidean 2-sphere, J. Nonlinear Sci. Appl 8, 2015, pp. 678-686.
[17] S. Izumiya, N. Takeuchi, Evolutoids and pedaloids of plane curves, Note Mat. 39, 2019, pp. 13-23.

[18] Y. Li and D. Pei, Pedal curves of frontals in the Euclidean plane, Math. Methods Appl. Sci. 41, 2018, pp. 1988-1997.

\section{Creative Commons Attribution License 4.0 (Attribution 4.0 International, CC BY 4.0)}

This article is published under the terms of the Creative Commons Attribution License 4.0

https://creativecommons.org/licenses/by/4.0/deed.en_US 